Research, Society and Development, v. 9, n. 10, e9719109400, 2020

(CC BY 4.0) | ISSN 2525-3409 | DOI: http://dx.doi.org/10.33448/rsd-v9i10.9400

\title{
Extravasamento de quimioterápicos: o papel do enfermeiro na emergência oncológica
}

Chemotherapic extravasation: the nurse's role in oncological emergency

Extravasación quimioterapica: el papel de la enfermera en la emergencia oncologica

Recebido: 18/10/2020 | Revisado: 25/10/2020 |Aceito: 26/10/2020 | Publicado: 29/10/2020

\section{Letícia Priscila Faria}

ORCID: https://orcid.org/0000-0003-0920-7369

Faculdade Cristo Rei, Brasil

E-mail: lepriscila02@ hotmail.com

Tatiane Renata Fagundes

ORCID: https://orcid.org/0000-0003-4634-360X

Faculdade Cristo Rei, Brasil

E-mail: tatiane@faccrei.edu.br

\section{Resumo}

O extravasamento de quimioterápicos antineoplásicos em via periférica, decorre de ações negligentes ou acidentais durante a administração da quimioterapia. O presente estudo teve por objetivo revisar a produção cientifica voltada a atuação do enfermeiro no manejo e na prevenção do extravasamento de quimioterápicos, por meio de uma revisão integrativa da literatura, nas bases de dados do Scielo e BVS, entre os anos de 2008 a 2020, mediante o emprego da combinação dos seguintes termos: enfermagem e quimioterapia; extravasamento de quimioterápicos; enfermagem oncológica; emergência oncológica. Foram obtidos 778 artigos, dos quais 19 (2,44\%) foram selecionadas, estes apontam que os enfermeiros possuem informações limitadas acerca dos riscos que envolvem o manuseio, a administração e o descarte de antineoplásicos, bem como as condutas após o extravasamento. Observamos que os enfermeiros que atuam no âmbito oncológico carecem de aprimoramento cientifico e formação adequada para desempenhar as atividades do setor, afim de minimizar os riscos de extravasamento e aprimorar a assistência fornecida ao paciente.

Palavras-chave: Câncer; Extravasamento de quimioterápicos; Emergência oncológica; Enfermagem oncológica. 


\begin{abstract}
The extravasation of antineoplastic chemotherapy drugs in the peripheral route, results from negligent or accidental actions during the administration of chemotherapy. The present study aimed to review the scientific production aimed at the nurse's performance in the management and prevention of the extravasation of chemotherapeutic agents, through an integrative literature review, in the Scielo and VHL databases, between the years 2008 to 2020, using the combination of the following terms: nursing and chemotherapy; extravasation of chemotherapeutic drugs; oncological nursing; oncological emergency. 778 articles were obtained, of which 19 (2.44\%) were selected, which point out that nurses have limited information about the risks that involve the handling, administration and disposal of antineoplastic agents, as well as the procedures after extravasation. We observed that nurses who work in the oncology field need scientific improvement and adequate training to perform the activities of the sector, in order to minimize the risks of leakage and improve the care provided to the patient.
\end{abstract}

Keywords: Cancer; Extravasation of chemotherapeutic drugs; Oncological emergency; Oncology nursing.

\title{
Resumen
}

La extravasación de fármacos quimioterápicos antineoplásicos en la vía periférica, resulta de acciones negligentes o accidentales durante la administración de quimioterapia. El presente estudio tuvo como objetivo revisar la producción científica dirigida al desempeño del enfermero en el manejo y prevención de la extravasación de agentes quimioterapéuticos, a través de una revisión integradora de la literatura, en las bases de datos Scielo y BVS, entre los años 2008 a 2020, utilizando la combinación de los siguientes términos: enfermería y quimioterapia; extravasación de fármacos quimioterápicos; enfermería oncológica; emergencia oncológica. Se obtuvieron 778 artículos, de los cuales se seleccionaron 19 $(2,44 \%)$, que señalan que los enfermeros tienen poca información sobre los riesgos que implican el manejo, administración y disposición de antineoplásicos, así como los procedimientos posteriores a la extravasación. Observamos que los enfermeros que laboran en el campo de la oncología necesitan un perfeccionamiento científico y una formación adecuada para el desempeño de las actividades del sector, con el fin de minimizar los riesgos de fugas y mejorar la atención brindada al paciente.

Palabras clave: Cáncer; Extravasación de fármacos quimioterápicos; Emergencia oncológica; Enfermería oncológica. 


\section{Introdução}

A incidência de câncer tem aumentado rapidamente ao redor do mundo, acompanhada pelo crescimento demográfico, envelhecimento da população, redução da mortalidade infantil e adoção de estilos de vida que aumentam o risco desta morbidade.

O câncer promove alterações no processo de divisão celular, resultando na proliferação rápida de células malignas (Souza, et al., 2017). Com objetivo de controlar tal patologia, diferentes tratamentos podem ser adotados, tais como cirurgia, transplante de medula, quimioterapia, radioterapia, imunoterapia, entre outro, variando entre o tipo da neoplasia, o avanço da doença e o órgão ou tecido envolvido (Correia, et al., 2011; Souza, et al., 2017).

A quimioterapia destaca-se como o método mais utilizado, devido à alta incidência de cura e ao aumento da sobrevida de pacientes em estágios avançados da doença. A administração de quimioterápicos (substâncias citotóxicas), ocorre majoritariamente pela administração por via intravenosa, contudo os aspectos vesicantes e irritantes destas drogas podem provocar edemas, escaras e a destruição tecidual, quando há o extravasamento (Moyses, et al., 2011; Radael, et al., 2016).

O extravasamento de quimioterápicos acontece devido a administração negligente ou acidental da quimioterapia no espaço perivascular ou no tecido subcutâneo e não nos vasos, causando lesões que variam de irritação local a necrose grave da pele, do tecido subcutâneo, do sistema vascular periférico, dos ligamentos, dos tendões e do tórax, contudo a gravidade do dano tecidual depende dos efeitos colaterais relativos à droga empregada e ao tempo de exposição, podendo comprometer a continuidade do tratamento e a qualidade de vida do paciente (Correia, et al., 2011; Radael, et al., 2016; Souza, et al., 2017).

Desta forma, faz-se necessária uma avaliação criteriosa da rede venosa e atenção aos possíveis sinais de extravasamento por parte do enfermeiro durante a aplicação, que devem estar capacitados para intervir de forma eficiente na prevenção do extravasamento, bem como na mitigação dos riscos e eventuais complicações durante a infusão (Correia, et al., 2011; Radael, et al., 2016; Souza, et al., 2017).

Nesta perspectiva, o presente estudo teve por objetivo revisar a produção cientifica voltada a atuação do enfermeiro no manejo e na prevenção do extravasamento de quimioterápicos. 


\subsection{Câncer e tratamentos}

A incidência de câncer no Brasil tem aumentado a cada ano, apresentando frequência similar a observada em países desenvolvidos, com cerca de 600 a 680 mil novos casos a cada ano, conforme as estimativas brutas e ajustadas do Instituto Nacional de Câncer (Inca, 2020). Para a região sul do Brasil foram estimados cerca de 114 mil novos casos de câncer em 2020, destes 35 mil (30\%) são esperados para o estado do Paraná (Inca, 2020). Estima-se que em 2020, no Brasil, o câncer será responsável por aproximadamente 224 mil mortes, 117 mil homens e 107 mil mulheres (Inca, 2020).

Segundo apresentado pela Organização Mundial de Saúde (OMS), o aumento acentuado de neoplasias ao redor do mundo, pode acarretar no adoecimento de 27 milhões de pessoas em 2030, levando 17 milhões a morte (OMS, 2017). No entanto, a incidência dos tipos de câncer varia amplamente, sendo regulada por diversos fatores: genéticos, nutricionais, ambientais e pelos hábitos de vida do indivíduo (uso de cigarro, drogas, bebidas e pratica de atividade física) (Fernandes \& Mello, 2010; Prado, 2014).

Caracterizado pelo crescimento celular descontrolado de células malignas, capazes de formar aglomerados celulares que podem originar tumores malignos, o câncer pode ocorrer em toda a extensão do corpo humano. São conhecidos cerca de 100 tipos diferentes de câncer, os quais muitas vezes podem ainda invadir outros tecidos e causar metástase (Bruno, et al., 2014; Prado, 2014).

No intuito de frear a mortalidade e melhorar a qualidade de vida dos pacientes, diversos tipos de tratamento para esta doença têm emergido nas últimas décadas, tais como: cirurgias, radioterapias, quimioterapias, imunoterapias, hormonioterapia, dentre outras. Estes devem ser realizados de forma planejada, combinada ou isolada, ao longo do tratamento, considerando as características do paciente, o estadiamento e a evolução da doença (Correia, et al., 2011; Souza, et al., 2017).

Tratamentos baseados em imunoterapias e/ou hormonioterapia, são recentes devido ao avanço dos estudos genéticos, porém destacam-se como uma alternativa relevante de tratamento para alguns tipos de câncer, mesmo com utilização ainda limitada. A hormonioterapia é caracteriza pela ingestão de inibidores hormonais, os quais são frequentemente usados no tratamento de cânceres de mama, no entanto tendem a promover elevado ganho de peso (Silva \& Dias, 2018). A imunoterapia, por sua vez, medeia o tratamento via utilização de antígenos tumorais, anticorpos e inibidores celulares, sendo 
comprovadamente eficiente em um número limitado de patologias, podendo desencadear diarreias severas que colocam em risco a vida do paciente (Kaliks, 2016).

Amplamente empregada no tratamento de cânceres, a radioterapia pode ser realizada antes ou após a intervenção cirúrgica, em tumores mais localizados e sensíveis a radiação ionizante. As chances de recidivas devem ser consideradas na escolha desse tratamento. Contudo, o paciente sofre durante o tratamento radioterápico com radiodermite e a mucosite, fatores que interferem na continuidade do tratamento e na qualidade de vida destes (Brateibach, et al., 2013).

A quimioterapia, por sua vez, utiliza-se de quimioterápicos que agem em fases diferentes do metabolismo celular, interferindo na síntese e transcrição de DNA, e/ou na produção de proteínas, afetando células malignas e benignas, produzindo diversos efeitos colaterais que diminuem a qualidade de vida do paciente durante o período das aplicações (Souza, et al., 2017). Contudo, está destaca-se pelos seus resultados positivos, como o prolongamento da vida de pacientes graves, sendo o tratamento mais utilizado por promover a maior incidência de cura para uma ampla diversidade de cânceres (Souza, et al., 2017; Correia, et al., 2011).

\subsection{Quimioterápicos e vias de administração}

Existe uma diversa gama de quimioterápicos, os quais possuem estruturas, funções e aplicações distintas, sendo classificados em dois grupos, o primeiro considera a estrutura química e a função em nível celular dos antineoplásicos, dividindo-os em seis subgrupos: agentes alquilantes; antimetabólitos; antibióticos antitumorais; plantas alcalóides; agentes múltiplos e hormônios, e antagonistas hormonais (Rang, Ritter, Flower \& Henderson, 2015).

Os agentes alquilantes, atuam a nível genético das células promovendo a alquilação do DNA e inviabilizando a divisão celular, tal mecanismo de ação é eficiente tanto no combate de células cancerosas em repouso, quanto em divisão ativa, porém a difusão do grupamento alquila na célula promove alteração em níveis celulares variados, conferindo toxicidade ao tratamento. Medicamentos como a isofofamida, ciclofosfamida, cisplatina e carboplatina, são exemplos de agentes alquilantes, empregados no controle do câncer (Maia, 2009; Silva \& Senna, 2010).

Os antimetabólicos possuem estrutura análoga a componentes importantes do ciclo celular como as bases nitrogenadas, purinas e pirimidinas, e o ácido fólico. Estes, atuam inibindo o avanço da síntese de DNA e/ou promovendo sequências erradas, decorrentes da 
incorporação indevida dos análogos de base nitrogenada. Além disso, os antimetabólitos, podem modificar a síntese de substâncias celulares e a produção de enzimas, afetando a divisão celular (Silva \& Senna, 2010).

Os antibióticos com atividade antitumoral, também atuam a nível genético, alterando e/ou impedindo a replicação e desprendimento das cadeias de RNA e DNA, são exemplos de antibióticos antitumorais a mitoxantrona, blemicina, doxorrubicina e a daunorrubicina (Silva \& Senna, 2010).

As plantas alcalóides impedem a mitose celular por interferir na distribuição cromossômica, culminando na morte da célula. São exemplos de alcaloides de plantas, o paclitaxel, a vincristina e a vimblastina, e outros compostos como o taxano e topotecano, que interferem na polarização da tubulina e na atividade da topoisomerase, respectivamente (Silva \& Senna, 2010).

Medicamentos como a asparginase, procarbazina e hidroxiuréia, são denominados de agentes múltiplos, por atuarem em diferentes níveis da duplicação e transcrição do material genético, levando a uma morte celular programada (Rang, Ritter, Flower \& Henderson, 2015).

Conforme mencionado anteriormente, os antineoplásicos são classificados ainda quanto a sua especificidade no ciclo celular, sendo denominados de específicos e nãoespecíficos. Os quimioterápicos específicos atuam em etapas particulares do ciclo celular e são mais eficientes no combate de tumores grandes de crescimento rápido, sendo os alcaloides e antimetabólitos exemplos desse grupo; já os antineoplásicos não-específicos, agem em qualquer fase do ciclo celular e sobre as todas as células, demandando de baixa divisão celular, tendo como exemplos os agentes alquilantes e antibióticos antitumorais (Maia, 2009).

Portanto, ao considerarmos o mecanismo de ação dos diferentes grupos de antineoplásicos a nível mitótico, meiótico, genético e metabólico, é possível compreendermos a elevada toxicidade que estes detêm, frente as células sadias do paciente, desencadeando uma série de efeitos adversos (Silva \& Senna, 2010).

A administração de drogas antineoplásicas pode ser realizada via oral, subcutânea, intrarterial, intravesical, intrapleural, intratecal, intraperitoneal, intramuscular e endovenosa (Bruno, et al., 2014; Inca, 2020; Schneider \& Pedrolo, 2011). Ademais, é importante que o enfermeiro compreenda a relação dos quimioterápicos com estas vias, para que conheça suas vantagens e desvantagens frente aos diferentes quadros clínicos.

Nesta perspectiva, a adoção da via oral para a administração de quimioterápicos é vantajosa, devido a facilidade e ao baixo custo, porém a ineficiência da absorção de alguns 
compostos pelo trato digestivo e os efeitos adversos no fígado, no estômago e intestino, limitam a adoção dessa via, são exemplos desses medicamentos o adrenocosticosteróides, metotexato, tioguanina, mercaptopurina, bussulfam e melfalan, (Inca, 2020; Maia, et al., 2010).

A administração por via subcutânea é responsável por aplicar o antineoplásico no tecido adiposo, possibilitando que este seja rapidamente absorvido pelos capilares, porém é pouco utilizada devido à alta toxicidade dos medicamentos; a asparaginase, é um exemplo de medicamento que pode ser administrado por essa via (Maia, et al., 2010).

A utilização da via arterial demanda da implantação cirúrgica do cateter, sendo acompanhada pelos fatores de risco relativos à utilização deste, porém possibilita a aplicação de altas doses de medicamento, combatendo mais ostensivamente o tumor e proporcionando menos efeitos colaterais ao paciente (Esteves, 2018).

A via intravesical promove o amplo contato da droga com a bexiga, sendo recomendada em tratamentos específicos por promover diversos problemas no trato urinário, como: cistite, infecções, alergias e contratura da bexiga (Inca, 2020).

A adoção da via intrapleural é interessante pois tende a prevenir a ocorrência de derrame pleural, por outro lado, a dor e a alta incidência de infecções decorrentes da implantação de dreno promove uma perda considerável na qualidade de vida do paciente, e ainda demanda complexidade nas aplicações necessitando de um aparato multiprofissional (Maia, et al., 2010).

A via intratecal, viabiliza que grandes quantidades do quimioterápico atinjam o liquido cérebro-espinhal, contudo, a punção lombar do medicamento demanda de enfermeiros habilidosos e especializados, os quais devem orientar e assistir corretamente o paciente, no intuito minimizar os problemas decorrentes do procedimento, como cefaleias, náuseas e vômito; os medicamentos administrados por essa via são a citarabina, dexametasona e o metotrexato (Maia, et al., 2010).

A via intramuscular proporciona a aplicação de grandes quantidades de medicamento e a rápida absorção pela corrente sanguínea nas regiões mais vascularizadas, sendo importante considerar as diluições necessárias e as dimensões corporais do paciente, bem como a idade, visto que em adultos para cada aplicação a dose não deve passar de $5 \mathrm{ml}$ e em crianças não deve ser maior que $3 \mathrm{ml}$, alternando os pontos de administração (Maia, et al., 2010).

No entanto, as propriedades séricas das drogas sugerem predominantemente que sua aplicação seja conduzida pela via endovenosa, a qual garante total absorção e bom efeito antineoplásico (Bruno, et al., 2014; Schneider \& Pedrolo, 2011). Os acessos venosos 
periféricos de maior calibre são os mais utilizados na administração dos quimioterápicos, apresentando um contexto complexo, visto que alguns pacientes podem manifestar problemas durante a aplicação, como irritações na pele, esclerose venosa, infecção, flebite e até necrose, fatores que interferem no avanço do tratamento (Schneider \& Pedrolo, 2011; Bruno, et al., 2014; Marquesin, et al., 2016).

\subsection{Problemas relacionados à quimioterapia}

A quimioterapia representa o tratamento mais eficiente no combate ao câncer, aumentando os índices de cura e conferindo maior expectativa de vida ao paciente; por outro lado, os quimioterápicos detêm elevada toxicidade, podendo ocasionar sintomas adversos no paciente, como diarreia, obstipação, náuseas, vômito, inapetência, lesões cutâneas, mucosite oral, formigamentos, tremores, perdas nutricionais, sensoriais, e locomotoras por afetar o equilíbrio (Guimarães, et al., 2015).

Os problemas dermatológicos são frequentes, estes podem ser locais ou sistêmicos, sendo a alopecia o mais incidente, seguida por alterações na unha, na pele, urticária, flebite, hiperpigmentação e prurido, afetando drasticamente a aparência e consequentemente a autoestima dos pacientes, privando-o do convívio social (Guimarães, et al., 2015; Pinho, Abreu \& Nogueira, 2016).

As lesões mais graves são majoritariamente relacionadas ao extravasamento de quimioterápicos, o qual pode provocar desde irritações cutâneas moderadas á necroses teciduais secundárias, as quais, geram dor severa e muitas vezes demandam de reconstrução tecidual via intervenção cirúrgica (Pinho, Abreu \& Nogueira, 2016). Tais intercorrências decorrentes da quimioterapia deixam os pacientes debilitados, sem vitalidade para desempenharem sua rotina, gerando um decréscimo na qualidade de vida e influenciando na eficácia, na continuidade do tratamento e consequentemente na cura (Pinho, Abreu \& Nogueira, 2016).

Os antineoplásicos atuam em diferentes etapas do ciclo celular interferindo a nível metabólico, genético e replicativo, apresentando baixa especificidade que promovem prejuízos tanto as células cancerosas, quanto as células normais, atribuindo alta toxicidade ao tratamento, a qual repercute em uma série de efeitos adversos que podem interferir a continuidade do tratamento com interrupção temporária ou definitiva (Caldeira \& GalbiattiDias, 2017; Silva \& Senna, 2010). 
A toxicidade hematológica destaca-se como o efeito adverso a mais perceptível ao longo do tratamento, sendo facilmente detectada por exames de sague de rotina, detectando nessas pacientes anemias, enquanto em casos mais grave levam a leucopenia e trombocitopenia (Maia, 2009).

Os danos cardíacos, por sua vez, manifestam-se mais tardiamente, devido ao caráter bioacumulativo dos quimioterápicos no organismo, promovendo insuficiência cardíaca. Já nos pulmões os antineoplásicos podem promover a tosse, taquipneia e dispneia (Amaral, 2019).

Outro efeito colateral frequente são as náuseas e vômitos, seguidos por estomatite e mucosite, decorrentes da toxicidade gastrintestinal das drogas, acompanhada ou não de prejuízos hepáticos, como icterícia, hepatite, necrose de células hepáticas, e dores abdominais, quadro que dificulta a alimentação do paciente, levado a quadros de anorexia que deixam o paciente fraco e debilitado (Caldeira \& Galbiatti-Dias, 2017; Silva, et al., 2019).

A toxicidade neurológica pode ser percebida já nos primeiros dias do tratamento, como formigamento, sonolência, perda do paladar, irritação, tontura, febre, rigidez na nuca, confusão, retornando durante as novas aplicações, ou tornando-se persistente ao longo do tempo; também são comuns manifestações de depressão, parestesias no pé, na mão e vertigem (Caldeira \& Galbiatti-Dias, 2017)

A nível reprodutivo, os antineoplásicos podem interferir no ciclo menstrual, via alterações na atividade ovariana e amenorreia, alterando a libido e o funcionamento testicular e ainda promover abortos e má formação nos fetos das gestantes em tratamento (Amaral, 2019).

A toxicidade renal pode ser rapidamente diagnosticada por meio da alteração dos níveis de ureia, ácido úrico e creatinina, promovendo prejuízos agudos ou crônicos nos pacientes, os quais tendem ser evitados pelo uso de diuréticos e por uma boa hidratação (Maia, 2009).

Os efeitos dermatológicos mais incidentes, decorrentes da toxicidade quimioterápica, são: alopecia, fotossensibilidade, alteração nas unhas, hiperpigmentação da pele, prurido, urticária e eritema. No entanto, os danos mais graves estão relacionados ao extravasamento venoso de quimioterápicos vesicantes, os quais promovem edemas graves nos pacientes (Rang, Ritter, Flower \& Henderson, 2015).

Portanto, são vários os efeitos adversos que os quimioterápicos podem manifestar nos pacientes, assim, cabe ao enfermeiro informar e orientar o paciente e a família sobre a possibilidade de tais intercorrências, bem como, sobre os exames que devem ser realizados para o acompanhamento do quadro clinico e as condutas recomendadas para melhorar a 
qualidade de vida durante esse período, como: alimentação, hidratação e repouso (Silva, et al., 2019).

\section{Metodologia}

A pesquisa foi realizada de forma descritiva, documental, por meio de uma revisão bibliográfica da literatura realizada nas bases de dados Scielo e BVS (Biblioteca Virtual em Saúde), de natureza qualitativa, mediante o emprego da combinação dos seguintes termos: enfermagem e quimioterapia; extravasamento de quimioterápicos; enfermagem oncológica; emergência oncológica.

A seleção de artigos seguiu alguns critérios: conter resumo e abordar o papel do enfermeiro no extravasamento de quimioterápicos, durante os anos de 2008 a 2020. Após a leitura flutuante dos artigos considerando os critérios apresentados, os artigos relevantes foram selecionados e seus dados tabulados (tabela 1). Posteriormente, os resultados foram organizados em duas categorias: $\left(1^{\circ}\right)$ informações sobre câncer, tratamentos, riscos incidentes do extravasamento de quimioterápicos; $\left(2^{\circ}\right)$ as condutas da equipe de enfermagem sobre o extravasamento de quimioterápicos e efeitos adversos e condutas de prevenção.

Tabela 1. Seleção de artigos.

\begin{tabular}{|c|c|c|c|}
\hline $\begin{array}{c}\text { BASE DE DADOS } \\
\text { ACESSADA }\end{array}$ & $\begin{array}{c}\text { PUBLICAÇÕES } \\
\text { OBTIDAS }\end{array}$ & $\begin{array}{c}\text { PUBLICAÇÕES } \\
\text { EXCLUÍDAS }\end{array}$ & $\begin{array}{c}\text { PUBLICAÇÕES } \\
\text { SELECIONADAS }\end{array}$ \\
\hline SCIELO & 376 & 367 & 9 \\
\hline BVS & 402 & 392 & 10 \\
\hline Total & 778 & 759 & 19 \\
\hline
\end{tabular}

Fonte: Os próprios autores.

\section{Resultados e Discussão}

Problemas na administração de quimioterápicos podem levar ao extravasamento das aplicações de drogas antineoplásicas em via periférica, promovendo danos locais, leves ou severos, que interferem na continuidade do tratamento e geram períodos longos de hospitalização (Correia, 2011; Bruno, et al., 2014), destacando-se como emergência oncológica por prejudicar consideravelmente a qualidade de vida do paciente. Contudo, este 
problema pode ser prevenido e/ou mitigado frente a assistência plena da enfermagem ao longo do tratamento quimioterápico (Souza, et al., 2017).

Estima-se que $90 \%$ das intervenções em ambulatórios oncológicos utilizam a vasopunção com dispositivo intravenoso periférico curto, o qual predispõe o paciente ao risco do extravasamento de quimioterápicos (Brito \& Lima, 2012). O extravasamento é caracterizado pelo derramamento da droga antineoplásica no tecido circunvizinho ao vaso sanguíneo, o qual pode ser proveniente do rompimento do vaso enfraquecido, do uso de veias baixo calibre, erro na punção, aplicação previa de tratamento radioterápico ou quimioterápico na região, doença vascular pré-existente, uso concomitante de medicamento, neuropatia periférica, e/ou de ações negligentes do profissional de enfermagem (Schneider \& Pedrolo, 2011).

Além disso, a proporção do dano decorre principalmente do tipo de medicamento utilizado no tratamento, estes são classificados em cinco categorias seguindo seu potencial de dano, sendo denominados respectivamente, como: vesicantes, esfoliantes, irritantes, inflamatórios e neutros. Sendo as drogas vesicantes e irritantes as mais frequentemente utilizadas (Schneider \& Pedrolo, 2011).

O extravasamento de drogas irritantes é caracterizado por provocar processos cutâneos dolorosos, com queimação, formação de edemas eritematosos e vesículas no local do derrame, com ausência de necrose tecidual (Brito \& Lima, 2012). Os processos inflamatórios aqui desenvolvidos são pontuais e temporários, de maneira contrária as demais drogas em que os danos são progressivos (Amorin, et al., 2020; Melo, et al., 2019).

As drogas vesicantes são altamente toxicas, capazes de produzir queimaduras químicas graves, são caracterizadas por provocar morte tecidual quando extravasados, são responsáveis por boa parte das emergências oncológicas graves, provocando infiltração, edemas, flebite, escaras, fatores que para serem mitigados demandam de conhecimento do enfermeiro (Souza, et al., 2017).

As complicações subsequentes ao extravasamento variam também frente a quantidade extravasada, o tecido afetado e o tempo de contato da droga com o tecido (Correia, et al., 2011). Os indícios de extravasamento podem ser detectados ainda durante a aplicação, mas também nas horas e dias subsequentes, sendo perceptíveis devido a sintomas como dor, desconforto local, queimação, irritação, inflamação, edema, eritema, enduração, ulceração e necrose (Rang, Ritter, Flower \& Henderson, 2015).

Tais complicações pioram consideravelmente a qualidade de vida do paciente, comprometendo a continuidade do tratamento e consequentemente suas chances de cura e a sobrevida, ressaltando assim a importância da identificação precoce do extravasamento pelo 
enfermeiro responsável, bem como a execução eficiente dos protocolos de cuidados fixados pela instituição (Chanes, Dias \& Gutierrez, 2008).

Estatisticamente, a incidência de extravasamento não é alta, os valores ficam entre $0,01 \%$ a $6,5 \%$ de todas as administrações de quimioterápicos, e os dados que são escassos (Melo, et al., 2019). No entanto, observando os diversos efeitos adversos da quimioterapia, como sepse, neutropenia, mucosite e distúrbios gastrintestinais, o número de extravasamentos se mostra significativo, por demonstrar grandes consequências a longo prazo, causando danos a nervos e até mesmo necessidade de cirurgia reconstrutiva, podendo ser ainda mais debilitante para o paciente do que a sua doença primária (Jackson-Rose, et al., 2017; Melo, et al., 2019).

A ocorrência de extravasamentos deve ser notificada em instrumento específico com data e hora da ocorrência, com dados sobre a droga usada e quantidade extravasada, localização do acesso venoso, tipo de dispositivo usado, calibre e número de punções, tipo de infusão e intervenções de enfermagem no momento da intercorrência (Schneider \& Pedrolo, 2011).

Um estudo retrospectivo dos anos de 2013 a 2015 realizado em um hospital universitário no interior do Estado de São Paulo buscou as notificações da ocorrência de extravasamento de agentes quimioterápicos, via sistema eletrônico. Em pacientes acima de 18 anos identificou-se 33 notificações de extravasamento por quimioterápicos; com maior incidência da droga Fluouroracil $(33,3 \%)$ e Paclitaxel $(18,2 \%)$ e as reações comuns foram o edema $(48,5 \%)$, dor $(12,1 \%)$ e hipersensibilidade $(9,1 \%)$. Os autores observaram que as notificações tinham dados incompletos, e possivelmente com subnotificações (Gozzo, Almeida \& Cruz, 2018).

Uma análise realizada no Centro de Assistência de Alta Complexidade de Oncologia (CACON) do Hospital Universitário Professor Alberto Antunes (HUPAA), no período de janeiro à dezembro de 2016 e de janeiro à agosto de 2017, mostrou a administração de 9051 infusões quimioterápicas com 16 episódios de extravasamentos de agentes antineoplásicos, dados considerados baixos (Silva, et al., 2018).

Uma análise do período de janeiro de 2009 a maio de 2012 em uma clínica Oncológica no interior do Estado de São Paulo demostrou que foram administradas 344 drogas vesicantes e 4432 drogas irritantes no período, total de 4776 infusões, com ocorrência de 2\% de extravasamentos com drogas irritantes (Irinotecano e Gemcitabina) em acesso venoso periférico e 1 extravasamento de droga vesicante Epirrubicina em cateter venoso central (Araki, et al., 2012). 
Em todos esses estudos analisados observamos que a ocorrência do extravasamento e sua identificação no momento da ocorrência, com ações imediatas para diminuir os danos, e acompanhamento domiciliar pós extravasamento são fatores que determinam os baixos números dessas ocorrências, além da uma equipe treinada para minimizar esses escapes.

O risco de extravasamento é considerado igual para todos os pacientes que recebem esse tipo de tratamento, porem de acordo com a literatura, algumas características de certos grupos tornem essa ocorrência mais comum em crianças e idosos, pelo fato da anatomia ser diferenciada nesses pacientes, com veias de menor calibre, frágeis e esclerosadas (Mendes, Morgado \& Morgado, 2013; Pikó, et al., 2013). Alguns locais anatômicos foram identificados com maior número de ocorrências de extravasamento, como o dorso da mão (33\%), a fossa antecubital (30\%) e o antebraço (20\%), havendo apenas 1 (2\%) registo de extravasamento associado ao cateter venoso central subcutâneo totalmente implantado (Silva, 2017).

Uma consulta a um banco de dados da clínica oncológica do Hospital Estadual da Criança (HEC), mostrou que 333 crianças/adolescentes com câncer submetidos à cateterização intravenosa periférica (CIP) nas unidades de ambulatório de oncologia a complicação mais presente foi o extravasamento, em 41,9\% dos casos (Santos et al., 2020).

A incidência de extravasamento na Central de Quimioterapia de Adultos e Infantil de um hospital universitário do interior do estado de São Paulo, variou de 0,0\% a 0,57\% para cateter periférico e foi de $0 \%$ para cateter totalmente implantado, no período de janeiro a julho de 2010, em concordância com os valores recomendados pela literatura (Moyses et al., 2011).

O Núcleo de Quimioterapia do Hospital AC Camargo Câncer Center tinha uma taxa de extravasamento, já considerada baixa de acordo com a literatura, 0,18\% antes de 2010. A partir desse ano, na tentativa de melhorar a eficácia do tratamento e segurança do paciente foi criado um protocolo que envolve a identificação do paciente com indicação de cateter venoso central, riscos associados a cada paciente, verificação do cateter central a cada 15 minutos, e outras verificações do local da punção. No ano de 2010 foram atendidos 20.040 pacientes para terapia antineoplásica e registrado o extravasamento de 36 casos $(0,18)$, em 2011 dos 22.080 pacientes atendidos, houve uma queda importante de extravasamento em $0,09 \%$, e no ano de 2012 foram atendidos 22397 pacientes, extravasamentos notificados: 17 com índice 0,07\%. A diminuição dos casos foi favorecida pelo protocolo de cuidados ao paciente de terapia antineoplásica, criado pelo hospital (Gasparini Junior, et al., 2013).

Ao longo deste estudo foram obtidas 778, das quais 19 (2,44\%) foram selecionadas. Estes abordaram os riscos que permeiam o tratamento quimioterápico e as condutas dos enfermeiros junto ao extravasamento de quimioterápicos, enfatizando a necessidade da 
Research, Society and Development, v. 9, n. 10, e9719109400, 2020

(CC BY 4.0) | ISSN 2525-3409 | DOI: http://dx.doi.org/10.33448/rsd-v9i10.9400

formação destes profissionais, bem como o estabelecimento de protocolos bem definidos e embasados em evidencias cientificas para minimizar os riscos de extravasamento e aprimorar a qualidade de vida dos pacientes (Tabela 2).

Tabela 2. Principais estudos utilizados para composição do presente estudo.

\begin{tabular}{|c|c|c|c|c|}
\hline $\begin{array}{c}\text { Base } \\
\text { de } \\
\text { dados }\end{array}$ & Nome do artigo & Autores & Objetivos & $\begin{array}{c}\text { Principais } \\
\text { resultados/conclusões }\end{array}$ \\
\hline Scielo & $\begin{array}{c}\text { A avaliação da rede } \\
\text { venosa pela } \\
\text { enfermagem em } \\
\text { mulheres } \\
\text { com câncer } \\
\text { ginecológico } \\
\text { durante o } \\
\text { tratamento } \\
\text { quimioterápico. }\end{array}$ & $\begin{array}{c}\text { Soares, } \\
\text { Almeida \& } \\
\text { Gozzo, } 2012 .\end{array}$ & $\begin{array}{l}\text { Avaliar a rede venosa } \\
\text { das mulheres com } \\
\text { câncer cervico-uterino } \\
\text { e analisar } \\
\text { intercorrências. }\end{array}$ & $\begin{array}{c}\text { Os resultados deste estudo } \\
\text { apontam para aspectos da prática } \\
\text { de enfermagem relacionados à } \\
\text { administração de quimioterápicos } \\
\text { e ressaltam a necessidade de } \\
\text { elaborar e implantar protocolos } \\
\text { para o cuidado. }\end{array}$ \\
\hline Scielo & $\begin{array}{l}\text { Emergência } \\
\text { oncológica: } \\
\text { atuação dos } \\
\text { enfermeiros no } \\
\text { extravasamento de } \\
\text { drogas } \\
\text { quimioterápicas } \\
\text { antineoplásicas }\end{array}$ & $\begin{array}{c}\text { Souza, et al., } \\
2017 .\end{array}$ & $\begin{array}{l}\text { Investigar a atuação } \\
\text { dos enfermeiros no } \\
\text { extravasamento de } \\
\text { quimioterápicos } \\
\text { antineoplásicos. }\end{array}$ & $\begin{array}{c}\text { Os enfermeiros evidenciaram } \\
\text { conhecimento quanto aos fatores } \\
\text { de risco, prevenção e } \\
\text { reconhecimento de sinais e } \\
\text { sintomas da ocorrência de } \\
\text { extravasamento por } \\
\text { quimioterápicos, mas não em } \\
\text { questões relacionadas a } \\
\text { classificação das drogas } \\
\text { antineoplásicas e intervenções em } \\
\text { emergências oncológicas. }\end{array}$ \\
\hline Scielo & $\begin{array}{c}\text { Prevenção e } \\
\text { conduta frente ao } \\
\text { Extravasamento de } \\
\text { agentes } \\
\text { antineoplásicos: } \\
\text { scoping review }\end{array}$ & $\begin{array}{l}\text { Melo, et al., } \\
2019 .\end{array}$ & $\begin{array}{l}\text { Identificar e sintetizar } \\
\text { as evidências } \\
\text { científicas sobre } \\
\text { prevenção e conduta } \\
\text { do extravasamento de } \\
\text { agentes } \\
\text { antineoplásicos em } \\
\text { pacientes adultos } \\
\text { realizado por } \\
\text { enfermeiros. }\end{array}$ & $\begin{array}{l}\text { É primordial a implementação de } \\
\text { protocolos baseados na evidência } \\
\text { científica sobre prevenção e } \\
\text { conduta diante do extravasamento } \\
\text { de antineoplásicos a fim de } \\
\text { fornecer segurança ao paciente e } \\
\text { respaldo à equipe de enfermagem. }\end{array}$ \\
\hline Scielo & Segurança do & Oliveira, et & Identificar e sintetizar & A segurança do paciente \\
\hline
\end{tabular}


Research, Society and Development, v. 9, n. 10, e9719109400, 2020

(CC BY 4.0) | ISSN 2525-3409 | DOI: http://dx.doi.org/10.33448/rsd-v9i10.9400

\begin{tabular}{|c|c|c|c|c|}
\hline & $\begin{array}{c}\text { paciente na } \\
\text { administração de } \\
\text { quimioterapia } \\
\text { antineoplásica e } \\
\text { Imunoterápicos } \\
\text { para tratamento } \\
\text { Oncológico: } \\
\text { scoping review. }\end{array}$ & al., 2019 & $\begin{array}{c}\text { as evidências } \\
\text { científicas sobre } \\
\text { segurança do paciente } \\
\text { oncológico na } \\
\text { administração de } \\
\text { quimioterápicos } \\
\text { antineoplásicos e } \\
\text { imunoterápicos }\end{array}$ & $\begin{array}{l}\text { oncológico na administração da } \\
\text { terapia antineoplásica ocorre } \\
\text { quando há a implementação de } \\
\text { protocolos, baseados em } \\
\text { evidências, educação permanente } \\
\text { dos enfermeiros e efetivação de } \\
\text { padrões e processos de segurança, } \\
\text { como estratégia para prevenção } \\
\text { de erros na administração dos } \\
\text { fármacos. }\end{array}$ \\
\hline Scielo & $\begin{array}{l}\text { Fatores de risco } \\
\text { para complicações } \\
\text { locais da terapia } \\
\text { intravenosa em } \\
\text { crianças e } \\
\text { adolescentes com } \\
\text { câncer }\end{array}$ & $\begin{array}{c}\text { Santos, et al., } \\
2020 .\end{array}$ & $\begin{array}{l}\text { Analisar fatores } \\
\text { preditores para a } \\
\text { incidência de } \\
\text { complicações } \\
\text { relacionadas a } \\
\text { cateteres venosos } \\
\text { periféricos em } \\
\text { crianças e } \\
\text { adolescentes com } \\
\text { câncer }\end{array}$ & $\begin{array}{c}\text { Incidência das complicações foi } \\
\text { de } 18,6 \% \text {. A modelagem das } \\
\text { variáveis confirmou que os } \\
\text { fatores de risco para } \\
\text { complicações em } \\
\text { crianças/adolescentes com câncer } \\
\text { foram: terapia intravenosa } \\
\text { periférica } \\
\text { prolongada, antecedentes de } \\
\text { complicações,medicamentos não } \\
\text { irritantes/vesicantes e soluções } \\
\text { vesicantes. }\end{array}$ \\
\hline
\end{tabular}

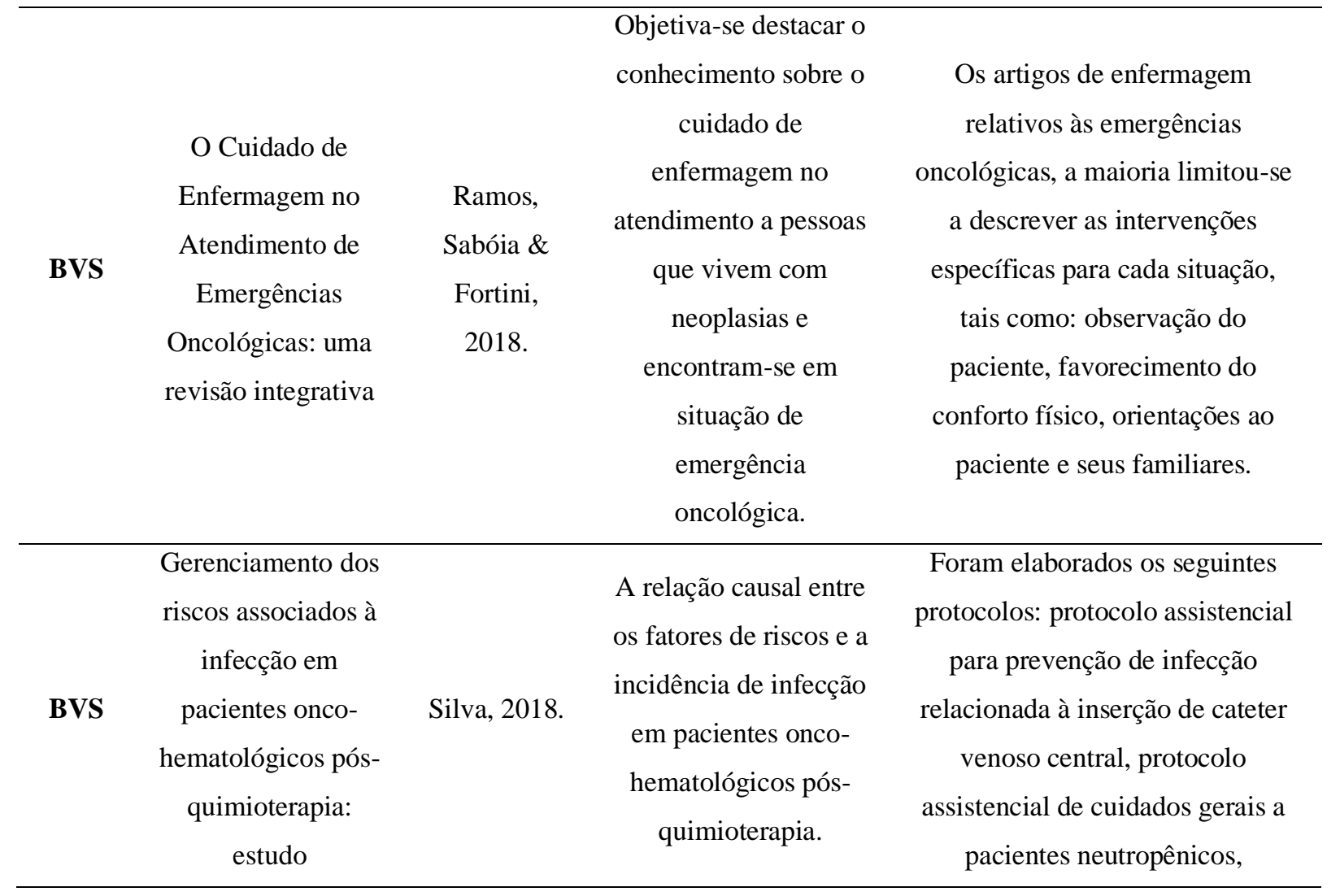


Research, Society and Development, v. 9, n. 10, e9719109400, 2020

(CC BY 4.0) | ISSN 2525-3409 | DOI: http://dx.doi.org/10.33448/rsd-v9i10.9400

observacional

Conhecimento dos

Profissionais de

Enfermagem sobre

BVS

Segurança do

Paciente

Oncológico em

Quimioterapia
Avaliar o

conhecimento dos

profissionais de

Costa, et al., enfermagem quanto à

2019.

segurança do paciente

oncológico em

tratamento

quimioterápico. protocolo assistencial para

prevenção de infecção

relacionada à mucosite oral e

protocolo assistencial para

prevenção de infecção

relacionada à transfusão de

concentrado de hemácias.

Há falhas de conhecimento nas

etapas do tratamento e

administração segura de

quimioterapia antineoplásica, na

identificação do paciente e na

comunicação eficaz. Observou-se

o empenho dos profissionais do

serviço em implementar o

Programa Nacional de Segurança

do Paciente (PNSP) com a

identificação dos pacientes com

crachá, prescrições impressas e

protocolos de extravasamento e

derramamento.

É necessária uma qualificação
Extravasamento de

trastuzumabe

BVS emtansina: manejo

em paciente

oncológico
Amorin, et

al., 2020.
Descrever o manejo

do extravasamento de

trastuzumabe

emtansina em uma

paciente com

carcinoma ductal

invasivo da mama. contínua da equipe, com base em

evidências científicas atualizadas,

para que ela possa intervir em

situações de extravasamento

quimioterápico de forma adequada.
Objetivou-se

descrever as situações

Lima-

Ribeiro, et

al., 2015.

negativas e positivas

experienciadas pelo

paciente em

tratamento oncológico

\section{Sequenciamento de}

infusão de

BVS
Mendonça,

et al., 2018.

contribuições para

a prática de

enfermagem
Identificar as

evidências científicas

disponíveis acerca das

interações

medicamentosas entre

antineoplásicos
Os pacientes com neoplasias ainda apresentam mais aspectos negativos do que positivos no decorrer do processo de adoecimento e tratamento. Interações farmacocinéticas e

farmacodinâmicas decorrentes da ordem de infusão, as quais subsidiaram a construção de um quadro determinando o melhor sequenciamento para cada uma 
Research, Society and Development, v. 9, n. 10, e9719109400, 2020

(CC BY 4.0) | ISSN 2525-3409 | DOI: http://dx.doi.org/10.33448/rsd-v9i10.9400

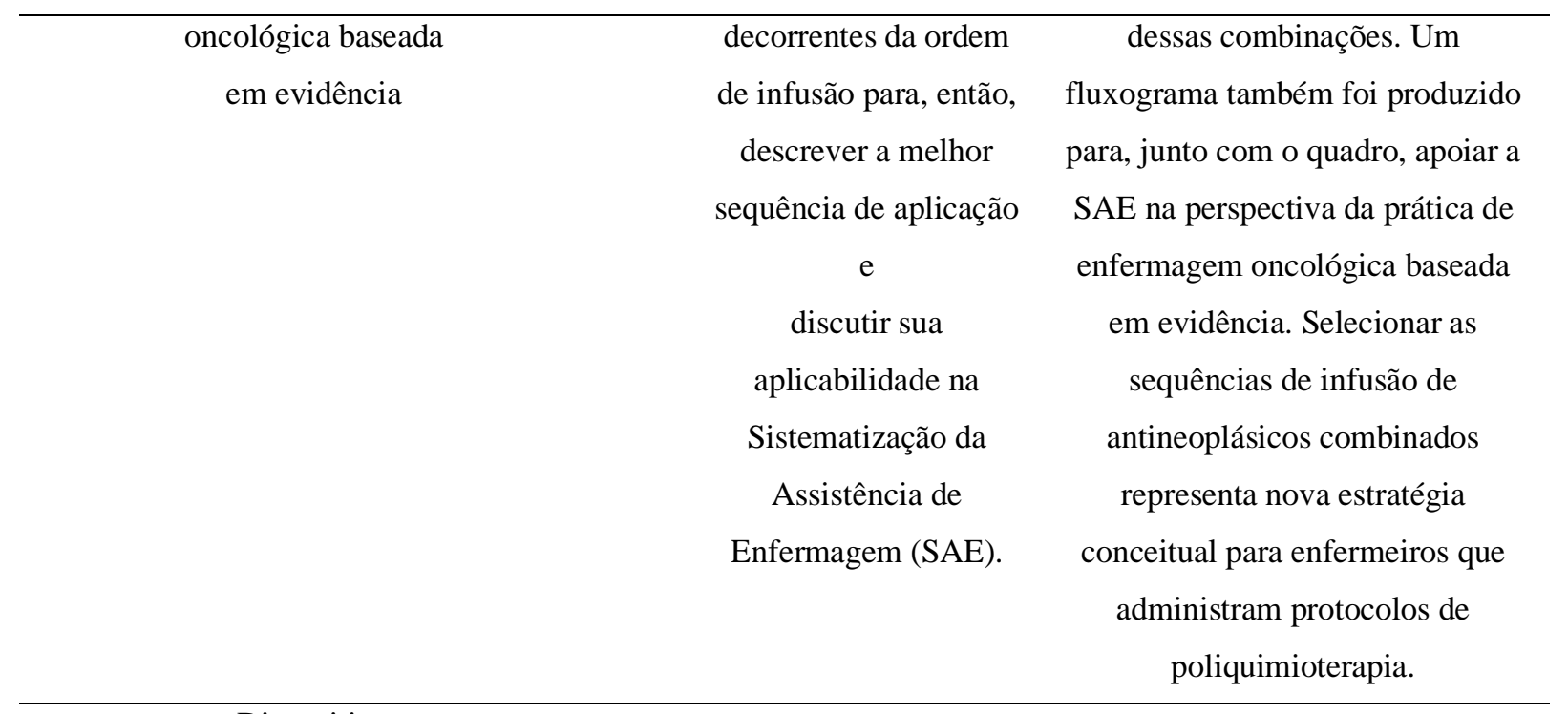

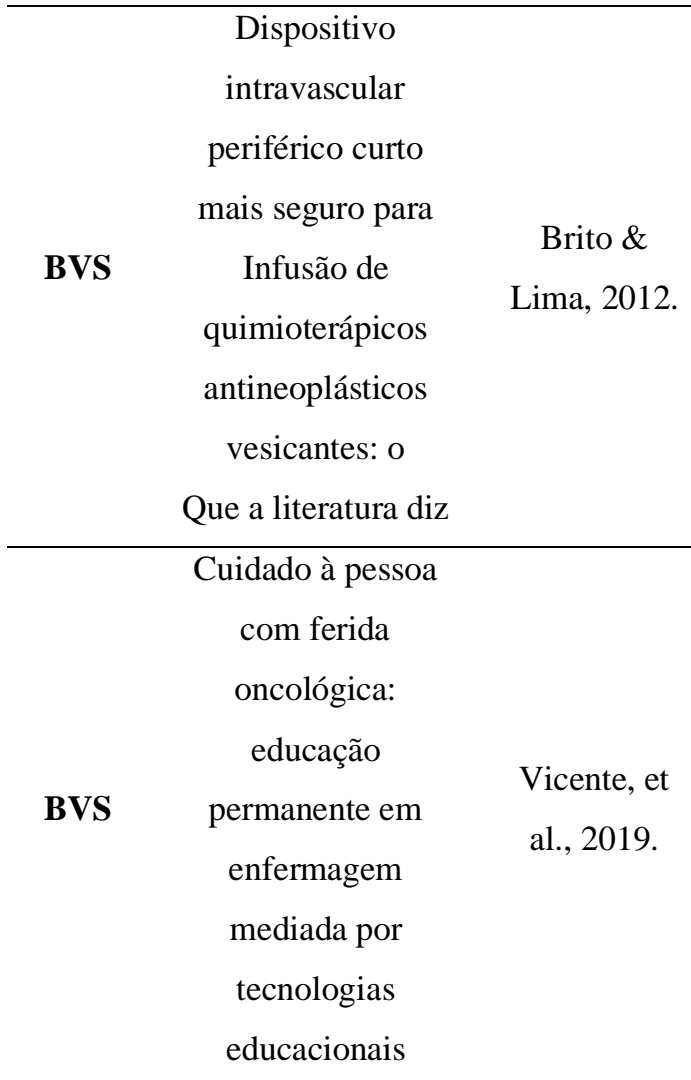

Prevenção da
ocorrência de
extravasamento,
principalmente, de
drogas
antineoplásticas
vesicantes.

Foi observado que não há consenso entre os autores quanto a indicação do dispositivo mais seguro para tal finalidade e conclui-se que vários fatores devem ser considerados para sua escolha.

Que a literatura diz 
Melo e colaboradores (2019), avaliaram bases de dados de diferentes países acerca das condutas dos enfermeiros especialistas em oncologia. Os estudos encontrados abordavam, majoritariamente, condutas de segurança preventiva ao extravasamento de quimioterápicos que são desempenhadas pelos enfermeiros. Os autores evidenciaram também, iniciativas desenvolvidas afim de sistematizar a assistência oncológica, bem como, as ações educativas e de aconselhamento desempenhadas pelo enfermeiro, sugerindo a padronização cientifica dos protocolos estipulados pelas instituições, frente as ações de prevenção e ação perante o extravasamento de drogas antineoplásicas.

Os enfermeiros oncológicos mostraram estar bem instruídos quanto os fatores de risco que permeiam o extravasamento de quimioterápicos, reconhecendo as estratégias de prevenção e tratamento, assim como os sintomas mais frequentes. Por outro lado, outros estudos apontam que os enfermeiros possuem informações limitadas acerca dos riscos que envolvem o manuseio, a administração e o descarte de antineoplásicos, evidenciando situações de risco ao longo dessas atividades, as quais podem favorecer a contaminação do ambiente (Baroni, et al., 2013; Ramos, Sabóia \& Fortini, 2018)

Silva-Rodrigues e colaboradores (2019), evidenciaram ainda que um número pequeno de enfermeiros que atuavam no setor oncológico era especializado na área. Estes destacaram ainda que o conhecimento que os profissionais detêm fora adquirido em cursos de capacitação executados por iniciativa própria, destacando a ausência de incentivo dos hospitais junto a formação dos enfermeiros que atuam nas emergências oncológicas.

As lacunas no conhecimento dos profissionais sobre a classificação das classes de antineoplásicos e os danos que cada qual pode desempenhar, findam por colocar o paciente em risco (Baroni, et al., 2013; Souza, et al., 2017; Ramos, Sabóia \& Fortini, 2018). Portanto, os enfermeiros devem conhecer o medicamento que será administrado, bem como sua classificação vesicante ou irritante, para que desempenhe corretamente as ações de prevenção e cuidado do extravasamento (Santos, et al., 2020; Ramos, Sabóia \& Fortini, 2018).

Corroborando com estas evidências, Costa e colaboradores (2019), descreveram fragilidades na assistência prestada pelos enfermeiros oncológicos, os quais não utilizam corretamente os EPIs, falham na higienização das mãos e no aconselhamento junto ao paciente e a família sobre o tratamento. Ademais, Brito e Lima (2012), evidenciaram a ausência de consenso quanto ao dispositivo médico mais seguro para minimizar as chances de extravasamento.

Tais limitações tendem a prejudicar as atividades desempenhadas por estes profissionais no tratamento do extravasamento, salientando a importância do estabelecimento 
de protocolos específicos para cada etapa da infusão, os quais devem orientar o enfermeiro, desde a escolha do acesso venoso, o aparato utilizado na punção e fixação do cateter, até as ações que devem ser executadas para cada tipo de droga extravasada (Soares, Almeida \& Gozzo, 2012; Silva, 2018).

Além do mais, a compreensão dos enfermeiros oncológicos sobre as características farmacocinéticas e farmacodinâmicas dos antineoplásicos, deve orienta-los quanto a ordem de infusão dos medicamentos (Mendonça, et al., 2018). Mendonça e colaboradores (2018), propuseram um fluxograma completo acerca das sequências de infusão das drogas quimioterápicas para protocolos de poliquimioterapia, afim diminuir os riscos ao paciente e otimizar o tratamento.

Oliveira e colaboradores (2019), ressaltaram em seu estudo que a implementação de protocolos aumenta a segurança do paciente durante a infusão dos antineoplásicos pelos enfermeiros, diminuindo também os riscos ocupacionais envolvidos, sendo necessário que os enfermeiros estejam em formação permanente, afim de que amparados por embasamento cientifico e por procedimentos assertivos aprimorem sua atividade, diminuindo a ocorrência de extravasamento (Senna, et al., 2013; Silva, 2018).

Frente aos erros dos enfermeiros na administração de quimioterápicos, a literatura sugere o desenvolvimento de revisões interdisciplinares das atividades e o investimento na formação dos profissionais (Oliveira, et al., 2019; Senna, et al., 2013). Considerando que o enfermeiro deve estar consciente das normas de biossegurança estipuladas pelo Inca e pelo Cofen para o manuseio e a aplicação de antineoplásicos, com vistas a minimizar os riscos provenientes de sua pratica (Senna, et al., 2013).

Lima-Ribeiro e colaboradores (2015), destacam em seu estudo a ausência de aspectos positivos pelos pacientes durante o tratamento do câncer. Os enfermeiros desempenham um convívio muito próximo com os doentes durante o tratamento, salientando a necessidade de um acompanhamento e/ou assistência eficaz pelos enfermeiros, para que o sofrimento dos pacientes seja amenizado e estes sejam fortalecidos via aprimoramento da qualidade de vida.

Dentre as ferramentas que o enfermeiro pode utilizar para melhorar a assistência prestada ao paciente, o emprego de novas tecnologias destaca-se, visto que estas possuem amplo acesso pela população e permitem um acompanhamento em tempo real dos sintomas dos pacientes, fortalecendo o elo estabelecido entre paciente-enfermeiro (Vicente, et al., 2019).

Conforme abordamos nos tópicos anteriores, embora a quimioterapia seja o tratamento mais empregado no combate de neoplasias malignas, há uma série de efeitos adversos 
decorrentes da sua administração, os quais podem ser instantâneos ou aparecerem gradativamente no decorrer das sessões (Souza, et al., 2017).

A enfermagem destaca-se neste contexto como responsável por orientar, acompanhar e assistir os pacientes oncológicos antes, durante e após a administração dos antineoplásicos, orientando e mitigando os problemas que surgem ao longo do percurso. Portanto, antes da administração do medicamento cabe ao enfermeiro que atua na emergência oncológica, orientar os pacientes e a família sobre os possíveis efeitos colaterais, bem como quais sintomas são normais e quais representam riscos. Neste momento o enfermeiro deve realizar orientações, acerca de como deve ser conduzido o repouso e a alimentação, afim de superar quadros de fraqueza e inapetência (Guimarães, et al., 2015).

Logo no primeiro contato com o paciente na sala de infusão, o enfermeiro é responsável por aconselhar e orientar o paciente e a família, acerca do tratamento quimioterápico. Conferindo instruções detalhadas sobre a infusão, as medicações que fazem parte do protocolo, e os efeitos colaterais de cada medicamento pode desencadear, alertando ainda sobre possíveis problemas que podem acontecer durante e após a aplicação venosa do tratamento (Amaral, 2019).

Ao longo do processo de aconselhamento, o enfermeiro oncológico deve considerar as crenças, a cultura e os hábitos de vida de cada indivíduo, afim de que os pacientes compreendam seu papel perante a realidade que estão sendo expostos, distanciando-se dos saberes instaurados pelo senso comum (Freitas, 2015; Silva, et al., 2019).

Ademais, é importante que durante o tratamento oncológico o enfermeiro acompanhe o paciente de forma integral. Neste sentido, algumas estratégias podem ser adotadas, para que os enfermeiros tenham um contato mais próximo dos pacientes, como telefonemas e o emprego de novas tecnologias para monitoramento, as quais podem ser utilizadas também como veículo de orientação, como o envio de material suplementar, como: cartilhas, textos e vídeos, que norteiem as doentes e as famílias frente ao tratamento quimioterápico realizado (Amaral, 2019).

Os enfermeiros criam vínculo com a família dos pacientes, educando e estabelecendo responsabilidades a estes durante o tratamento. Desta forma, o enfermeiro oncológico deve superar a sua pratica profissional ambulatorial e gerenciar a evolução do paciente ao longo do tratamento, enfatizando a importância de determinadas posturas para a melhoria na qualidade de vida do paciente (Silva, et al., 2019).

O enfermeiro é responsável também por checar a prescrição médica da droga empregada no tratamento, a dosagem, a via e o tempo de administração, bem como o histórico 
alérgico do paciente e os exames recentes, aplicando antiemético, quando necessário, no intuito de prevenir eventuais problemas (Guimarães, et al., 2015; Souza, et al., 2017).

Posteriormente a realização da quimioterapia o enfermeiro deve assistir periodicamente o paciente no manejo dos sintomas, examinando se o antiemético está sendo efetivo, se o paciente está hidratado, se há dor ou desconforto, se houve perda de peso e se há presença de mucosite na cavidade oral, registrando os efeitos adversos e realizando encaminhamento médico quando necessário (Guimarães, et al., 2015).

Estudos apontam que a incidência de extravasamento em diversos hospitais do mundo, se deve ao emprego concomitante de tratamentos na mesma região e principalmente de erros dos profissionais de enfermagem. Portanto, para minimizar os impactos do tratamento, as instituições de saúde devem estabelecer medidas preventivas claras por meio de protocolos de gerenciamento de biossegurança bem definidos e enfermeiros capacitados (Moyses, et al., 2011; Souza, et al., 2017).

Desta forma, a formação do enfermeiro que atua no setor oncológico deve prepará-lo para as peculiaridades do tratamento quimioterápico, visto que, este profissional deve estar atento tanto na escolha do dispositivo utilizado no momento da punção, quanto no vaso sanguíneo a ser puncionado, evitando veias demasiadamente frágeis e endurecidas e locais que sofreram muitas punções, considerando ainda o tipo de medicamento de cada tratamento (Schnerder \& Pedroso, 2011; Moyses, et al., 2011).

Ao longo da administração do tratamento o enfermeiro deve comportar-se de forma vigilante, verificando constantemente o fluxo da infusão, a ausência de retorno venoso, as queixas dos pacientes, irritações e edemas no local da punção. Tais checagens devem acontecer entre um intervalo de 10 a 20 minutos, variando de acordo com a duração da aplicação. Ao identificar algum sintoma a aplicação deve ser suspensa imediatamente e o liquido infiltrado no dispositivo de punção deve ser aspirado, o local lavado, e o membro afetado pode ser elevado, para que haja diminuição do potencial do edema. Concomitantemente, devem ser realizadas complexas frias ou quentes conforme o medicamento extravasado (Chanes, Dias \& Gutierrez, 2008; Rodrigues, et al., 2012; Radael, et al., 2016).

Assim, é fundamental que os enfermeiros sejam capacitados durante sua formação inicial e continuada para atuar de forma eficiente frente as emergências oncológicas, como o extravasamento, atuando também na prevenção e identificação rápida das complicações relacionadas ao extravasamento, diminuindo o sofrimento do paciente (Radael, et al., 2016; Vicente, et al., 2019). 
No intuito de regulamentar as ações do enfermeiro após o extravasamento é necessário é este esteja ciente que a severidade do dano está relacionada com aos aspectos físicoquímicos da droga vesicante, tal como $\mathrm{pH}$, osmolaridade e peso molecular, e também com a volume extravasado e o tempo de contato com o tecido (Chanes, Dias \& Gutierrez, 2008).

Portanto, para mitigar os prejuízos do paciente, é crucial que após o extravasamento o enfermeiro siga rigorosamente as instruções do Procedimento operacional padrão (POP), de sua instituição, o qual deve trazer de forma sistematizada as condutas recomendadas. Bruno e colaboradores (2014), desenvolveram em seu trabalho um modelo de POP para nortear as ações dos profissionais de enfermagem após o extravasamento. Destacando a necessidade de: interrupção imediata da aplicação, utilização de antidoto, remoção do dispositivo de aplicação, realização de curativos oclusivos estéreis e compressas, além de orientar o paciente sobre a necessidade de deixar o membro elevado por 48 horas, administrar analgésico, anotar no prontuário e comunicar o médico.

O acompanhamento da lesão pelo enfermeiro nas primeiras 24 ou 48 horas após o extravasamento é fundamental para que os danos sejam minimizados, uma vez que os primeiros sinais podem demorar a se manifestar acarretando em um dano tecidual progressivo e severo. Ao longo deste acompanhamento aspectos locais como cor da pele, temperatura, dor, febre, integridade tecidual, mobilidade e presença de edema devem ser continuamente avaliados (Chanes, Dias \& Gutierrez, 2008; Souza, et al., 2017).

Ademais, o tipo da droga extravasada deve ser rigorosamente considerado na adoção do tratamento, visto que para cada tipo de droga é recomendado um tipo de compressa (quente ou fria), em um determinado intervalo de tempo, bem como um antidoto especifico que deve ser empregado com apoio médico (Bruno, et al., 2014).

$\mathrm{O}$ extravasamento de quimioterápicos afeta a qualidade e a expectativa de vida do paciente, portanto, deve ser gerenciado de forma séria e eficaz pelos enfermeiros, para que os danos sejam minimizados e a saúde restabelecida (Souza, et al., 2017; Radael, et al., 2016).

\section{Considerações Finais}

Concluímos que o conhecimento dos enfermeiros oncológicos acerca dos riscos e condutas, junto ao manuseio e o extravasamento de quimioterápicos antineoplásicos são limitados, refletindo em ações negligentes perante as medidas de biossegurança, as quais expõe profissionais e pacientes a riscos. As lesões do extravasamento de antineoplásicos vesicantes podem promover necrose tecidual, as quais são agravadas pela falta de preparo dos 
enfermeiros na contenção da lesão, interrompendo o tratamento quimioterápico e prejudicando a qualidade de vida do paciente.

Sugerimos que os enfermeiros que atuam em emergências oncológicas detenham formação adequada para desempenhar as atividades do setor, afim de que assim minimizem os riscos de extravasamento e aprimorem a assistência fornecida ao paciente.

\section{Referências}

Amaral, L. B. D. M. X. (2020). Cuidado de enfermagem nos efeitos adversos na quimioterapia para câncer de mama: revisão narrativa da literatura. Monografia. Universidade Federal do Tocantins.

Amorim, B. F., Kameo, S. Y., Silva, G. M., Hardman, G. L., Costa, J. D. S., \& Ramos, M. J. O. (2020). Extravasamento de trastuzumabe emtansina: manejo em paciente oncológico. Rev. enferm. UFPE on line, 1-9.

Araki, A. P. et al. Análise do índice de extravasamento e cuidados de Enfermagem em uma clínica privada Congresso de Cancerologia - CONCAN, 2012. Recuperado de $<$ https://www.grupooncoclinicas.com/inorp/nossos-resultados/analise-do-indice-deextravasamento-e-cuidados-de-enfermagem-em-uma-clinica-privada/> .

Baroni, F. C. A. L., Oliveria, J. C. D. M., Guimarães, G. D. L., Matos, S. S. D., \& Carvalho, D. V. (2013). O trabalhador de enfermagem frente o gerenciamento de resíduo químico em unidade de quimioterapia antineoplásica. REME rev. min. enferm, 554-559.

Brateibach, V., Domenico, E. L. B., Berlezi, E. M., Loro, M. M., Rosanelli, C. L. S. P., Gomes, J. S., \& Kolankiewicz, A. C. B. (2013). Sintomas de pacientes em tratamento oncológico. Revista Ciência \& Saúde, 6(2), 102-9.

Brito, C. D. D., \& Lima, E. D. R. D. P. (2012). Dispositivo intravascular periférico curto mais seguro para infusão de quimioterápicos antineoplásticos vesicantes: o que a literatura diz. Revista Mineira de Enfermagem, 16(2), 275-279. 
Bruno, M. L. M., Barbosa, I. M., Sales, D. S., de Menezes, A. V. B., Gomes, A. F., \& Alves, M. D. S. (2014). Condutas de enfermagem no extravasamento de quimioterápicos antineoplásicos: protocolo operacional padrão. Journal of Nursing UFPE/Revista de Enfermagem UFPE, 8(4).

Caldeira, b. M., \& Galbiatti-dias, a. L. S. (2017). Acompanhamento e avaliação dos efeitos adversos da quimioterapia em pacientes com câncer de mama. Revista Corpus Hippocraticum, 1(1).

Chanes, D. C., Dias, C. G., \& Gutiérrez, M. G. R. (2008). Extravasamento de drogas antineoplásicas em pediatria: algoritmos para prevenção, tratamento e seguimento. Revista Brasileira de Cancerologia, 54(3), 263-273.

Correia, J. N., Albach, L. S. P., \& Albach, C. A. (2011). Extravasamento de quimioterápicos: conhecimentos da equipe de enfermagem. Revista Ciência \& Saúde, 4(1), 22-31.

Costa, A. G., Costa, M. S. C. R., da Silva Ferreira, E., de Sousa, P. C., dos Santos, M. M., Lima, D. E. D. O. B., \& Ramos, A. M. P. C. (2019). Conhecimento dos Profissionais de Enfermagem sobre Segurança do Paciente Oncológico em Quimioterapia. Revista Brasileira de Cancerologia, 65(1).

Fernandes, I. C., \& Mello, A. A. (2010). Entendendo e combatendo o câncer. TEMA-Revista Eletrônica de Ciências (ISSN 2175-9553), 7(10/11).

Freitas, K. A. B. S. (2015). Estratégias para a administração segura de antineoplásicos [dissertação]. Botucatu (BR): Universidade Estadual Paulista, Faculdade de Medicina de Botucatu.

Gasparini Junior, J. L. et al. Extravasamento de quimioterápico-melhorias para garantia da segurança do paciente. [s.l: s.n.].

Gozzo, T. O., de Almeida, T. D., \& da Cruz, L. A. P. (2018). < b> Notificação de extravasamento de agentes quimioterápicos em um hospital universitário-Notification of 
extravasation of chemotherapeutic agents in a university hospital $<$ b. Ciência, Cuidado $e$ Saúde, 17(2).

Guimarães, R. D. C. R., Gonçalves, R. P. F., de Almeida Lima, C., Torres, M. R., \& de Oliveira, C. S. (2015). Ações de enfermagem frente às reações a quimioterápicos em pacientes oncológicos. Revista de Pesquisa Cuidado é Fundamental Online, 7(2), 2440-2452.

Instituto Nacional do Câncer- INCA (2020). Recuperado de: https://www.inca.gov. br/numeros-de-cancer.

Jackson-Rose RN, B. S. N., Groman, A., Dial, L. S., Leah Atwell, M. S. N., Judy Graham, M. S., Semler, R. O. N., \& Eileen Costantinou, M. S. N. (2017). Chemotherapy Extravasation: establishing a national benchmark for incidence among Cancer Centers. Clinical Journal of Oncology Nursing, 21(4), 438.

Kaliks, R. A. (2016). Avanços em oncologia para o não oncologista. einstein (São Paulo), 14(2), 294-299.

Lima Ribeiro, S., Schwartz, E., Feijó, A. M., dos Santos, B. P., Garcia, R. P., \& Lise, F. (2015). Incidentes críticos experienciados no tratamento da doença oncológica. Revista de Enfermagem do Centro-Oeste Mineiro, 5(3).

Maia, P. G. (2009). A atividade da equipe de enfermagem e os riscos relacionados à exposição a quimioterápicos antineoplásicos no setor de oncologia de um hospital público do estado do Rio de Janeiro. (Doctoral dissertation).

Maia, V. R. (2010). Protocolos de Enfermagem. Administração de quimioterapia antineoplásica no tratamento de hemopatias malignas. Hemório.

Marquesin, A. P., Campanaro, M. P., \& Herr, G. E. G. (2016). Extravasamento de quimioterápicos e a equipe de enfermagem. Relato de experiência. Evento: XVII Jornada de Extensão da Unijuí. 
Melo, J. M. A., Oliveira, P. P. D., Souza, R. S., Fonseca, D. F. D., Gontijo, T. F., \& Rodrigues, A. B. (2019). Prevention and conduct against the Extravasation of antineoplastic chemotherapy: a scoping review. Revista Brasileira de Enfermagem, 73(4).

Mendes, M. D. R. (2013). Normas de atuação em caso de extravasão de citotóxicos injetáveis: experiência profissionalizante na vertente de farmácia comunitária, hospitalar e investigação (Doctoral dissertation).

Mendonça, A. B., Pereira, E. R., Magnago, C., Barreto, B. M. F., Goes, T. R. P., \& Silva, R. M. C. R. A. (2018). Sequenciamento de infusão de antineoplásicos: contribuições para a prática de enfermagem oncológica baseada em evidência. Revista Eletrônica de Enfermagem, 20.

Moysés, A. M. B., SÁ, L., Vanzo, D. S. D., Pavan, F. A. G., Borges, M. S., \& Machado, S. H. (2011). Índice de extravasamento de quimioterapia antineoplástica em cateter venoso periférico e totalmente implantado. Seção de Enfermagem da Central de Quimioterapia e Oncologia, (2).

Oliveira, P. P. D., Santos, V. E. P., Bezerril, M. D. S., Andrade, F. B. D., Paiva, R. D. M, \& Silveira, E. A. A. D. (2019). Segurança do paciente na administração de quimioterapia antineoplásica e de imunoterápicos para tratamento oncológico: revisão do escopo. Texto \& Contexto- Enfermagem, 28.

OMS. Organização Mundial da Saúde. Guide to cancer early diagnosis, Geneva: World Health Organization; 2017. Recuperado de https://canceratlas.cancer.org/about/

Pikó, B., Laczó, I., Szatmári, K., Bassam, A. A, Szabó, Z., Ócsai, H. H, \& Csotye, J. J (2013). Visão geral da gestão de extravasamento e possibilidades de redução de risco com base em dados da literatura. Journal of Nursing Education and Practice, 3 (9), 93.

Pinho, M. S., Abreu, P. A., \& Nogueira, T. A. (2016). Atenção Farmacêutica a pacientes oncológicos: uma revisão integrativa da literatura. Revista Brasileira de Farmácia Hospitalar e Serviços de Saúde, 7(1). 
Prado, B. B. F. D. (2014). Influência dos hábitos de vida no desenvolvimento do câncer. Ciência e Cultura, 66(1), 21-24.

Radael, W., Radael, W., Carvalho, A., Dutra, J., Lazarone, B., \& Fumian, L. (2016). Avaliação do risco de extravasamento de quimioterápico antineoplásico administrado via cateter de inserção periférica: relato de caso. Acta Biomedica Brasiliensia, 7(1), 124-129.

Ramos, L. G. A., Sabóia, V. M., \& Fortini, R. G. (2018). O Cuidado de Enfermagem no Atendimento de Emergências Oncológicas: uma revisão integrativa. Revista Enfermagem Atual In Derme, 86(24).

Rang, R., Ritter, J. M., Flower, R. J., \& Henderson, G. (2015). Rang \& Dale Farmacologia. Rio de Janeiro: Elsevier Brasil.

Rodrigues, C. C., Guilherme, C., Costa Júnior, M. L. D., \& Carvalho, E. C. D. (2012). Fatores de risco para trauma vascular durante a quimioterapia antineoplásica: contribuições do emprego do risco relativo. Acta Paulista de Enfermagem, 25(3), 448-452.

Santos, L. M. D., Silva, C. S. G., Machado, E. S., Almeida, A. H. D. V., Silva, C. A. L. D., Silva, B. S. M., \& Avelar, A. F. M. (2020). Fatores de risco para complicações locais da terapia intravenosa em crianças e adolescentes com câncer. Revista Brasileira de Enfermagem, 73(4).

Schneider, F., \& Pedrolo, E. (2011). Extravasamento de drogas antineoplásicas: avaliação do conhecimento da equipe de enfermagem. Revista Mineira de Enfermagem, 15(4), 522-529.

Silva, A. R. Á. A. (2017). Intervenção do enfermeiro especialista na prevenção e monitorização de extravasamento de citostáticos. Dissertação de mestrado: Escola superior de Enfermagem de Lisboa.

Silva, L. C. A., Signor, A. C., Pilati, A. C. L., Dalfollo, B. R., \& Oliveira, D. R. (2019). Abordagem Educativa ao Paciente Oncológico: Estratégias para Orientação acerca do Tratamento Quimioterápico. Revista Brasileira de Cancerologia, 65(1). 
Silva, L. D. B. D. (2018). Gerenciamento dos riscos associados à infecção em pacientes oncohematológicos pós-quimioterapia: estudo observacional. Tese. Universidade Federal Fluminense.

Silva, R. C. F. (2017). Guide to Cancer Early Diagnosis. Revista Brasileira de Cancerologia, 63(1), 41-42.

Silva-Rodrigues, F. M., da Silva, J. K., Nunes, M. D. R., dos Santos Cardoso, L. G., \& Nascimento, L. C. (2019). Atitudes de enfermeiros na administração de quimioterápicos em oncologia pediátrica. Revista Enfermagem UERJ, 27, 37458.

Soares, C. R., Almeida, A. M. D., \& Gozzo, T. D. O. (2012). Evaluación de la red venosa por la enfermería en mujeres con cáncer ginecológico durante el tratamiento de quimioterapia. Escola Anna Nery, 16(2), 240-246.

Souza, N. R. D., Bushatsky, M., Figueiredo, E. G. D., Melo, J. T. D. S., Freire, D. D. A., \& Santos, I. C. R. V. (2017). Emergência oncológica: atuação dos enfermeiros no extravasamento de drogas quimioterápicas antineoplásicas. Escola Anna Nery, 21(1).

Vicente, C., Amante, L. N., Santos, M. J. D., Alvarez, A. G., \& Salum, N. C. (2019). Cuidado à pessoa com ferida oncológica: educação permanente em enfermagem mediada por tecnologias educacionais. Revista Gaúcha de Enfermagem, 40.

\section{Porcentagem de contribuição de cada autor no manuscrito}

Letícia Priscila Faria- 60\%

Tatiane Renata Fagundes- 40\% 